\title{
Pharmacotherapy Patient Counselling: Another feather in the cap for Clinical Pharmacology discipline in a resource-limited setting
}

Amol Patil ${ }^{1}$, CVN Harish ${ }^{1}$, Samiksha Bhattarjee ${ }^{1}$, Ankit Kumar ${ }^{1}$, and Vishal Sharma ${ }^{2}$

${ }^{1}$ Post Graduate Institute of Medical Education and Research

${ }^{2}$ PGIMER

October 26, 2020

Title page

Type of manuscript: Letter to editor

Title: Pharmacotherapy Patient Counselling: Another feather in the cap for Clinical Pharmacology discipline in a resource-limited setting

Authors: Amol N Patil1, CVN Harish1, Samiksha Bhattacharjee1, Ankit Kumar1, Vishal Sharma2

1Department of Pharmacology, PGIMER, Chandigarh, India

2Department of Gastroenterology, PGIMER, Chandigarh, India

Corresponding Author:

Dr Amol N Patil

Associate Professor

Department of Pharmacology

Postgraduate Institute of Medical Education \& Research

Chandigarh 160012, India

Phone: 0091-172-2755244 (O)

Mobile: +91-9990245973, +91-7087008957

Email: patil.amol@pgimer.edu.in, amolpatilpgi@yahoo.com

Total number of pages: 2

Word counts of main text: 740 (excluding the references, tables),

Word counts for the abstract : NA

Total number of tables : 0

Total number of figures: 1

Source(s) of support : Nil

Conflict of interest : Nil 


\section{Acknowledgement: None}

\section{IRB approval status: Approved}

Key words : Clinical Pharmacology, developing world, pharmacotherapy counselling

\section{Pharmacotherapy Patient Counselling: Another feather in the cap for Clinical Pharmacology discipline in a resource-limited setting}

The clinical Pharmacology discipline came into existence in India 50 years back. ${ }^{1}$ The scope of clinical Pharmacology is continuously expanding round the globe. There is a parallel rise in patient-oriented services such as Evidence-based medicine on-call service- an advanced version of the drug information center (DIC) service along with many more other services. ${ }^{2}$ Few of these services are routinely taken up as a part of the $\mathrm{DM}$ (Doctor of medicine) clinical pharmacology curriculum in India to train resident doctors. The current curriculum of the super specialization DM clinical Pharmacology in India is not uniform throughout the country and varies as per the resources available. ${ }^{1}$ In the western side of the globe, the hospital pharmacists and clinical pharmacists are actively helping patients with drug therapy related counseling over and above clinicians offering an ample amount of time for a thorough discussion over disease prognosis and treatment courses. Indian scenario is almost opposite to that of the western one due to several reasons. Disproportionate doctor-patient ratio, daily wages lost in waiting for consultation in public hospitals, scanty amount of time spent per patient consultation, absence of dedicated clinical pharmacist designation, are some of the major

hurdles in providing quality pharmacotherapy with proper counseling in the country. ${ }^{3}$ Thus, there is a call for the novel clinical pharmacology service via which one can bridge this gap and help the local population with relevant counseling related to the treatment plan and tailor it towards patient-specific concerns. ${ }^{4}$

Authors hypothesized that difficult counseling scenarios such as drugs to be avoided in mastocytosis patients, myasthenia gravis, long QT syndrome, porphyria patients, could be the arena to explore for pharmacology and clinical pharmacology departments in developing countries. With this background idea, the DIC started patient counseling sessions for the five diseases (Figure 1). The clinician colleagues were approached with a pre-designed message via the central mobile SMS service of our institution. The text message included "For any pharmacotherapy counseling please send your patients to the drug information center, Department of Pharmacology. The patient will be guided regarding drugs to be avoided and how to stratify the risk associated with drugs (prescription and over the counter) used in the following five disorders- G6PD(glucose-6phosphate dehydrogenase) deficiency, Long QT syndrome, Mastocytosis, Myasthenia gravis, and Porphyria". The DIC received 97 patients in six months(August2019-January2020). The patients were requested for feedback post counseling session on a questionnaire with eight multiple choice questions(supplementary file table 1). Institutional ethics committee permission was obtained before initiation of pilot run service. Ninety one $(93.8 \%)$ were satisfied with the length and discussion whereas six patients $(6.18 \%)$ were not. Efforts were taken to identify etiology for the unsatisfactory response. Three patients reported long waiting time was the reason. On further questioning whether they received counseling in a suitable time of day or not, two patients reported that they received drug therapy counseling within a suitable time while one patient didn't agree. Fisher's exact test showed no statistically significant difference between the satisfaction rating versus a suitable or unsuitable time frame of counseling. Fisher's exact value was 0.5 and a non-significant result at p-value 0.05. Another attempt was made to check the association of the educational status of patients and the overall rating. Out of total 97 patients, 23 were postgraduate, 30 were graduate, 42 had completed schooling and two had not. One unsatisfactory response was received from postgraduate and graduate patients each. Fisher exact test applied on educational status versus satisfaction rating showed a non-significant difference at p-value 0.05 with Fisher exact value of one. Efforts were also made to check whether the resident on duty was able to communicate and answer the queries raised by the patients satisfactorily. One patient $(1.03 \%)$ giving an unsatisfactory rating said that patient's queries were resolved appropriately but beyond a suitable time frame. A similar study conducted by our group on doctor's feedback previously showed that more than $98 \%$ of clinicians found the service satisfactory. There was no significant association between the clinician's academic experience and the satisfaction rating. Over and above more than $96 \%$ of clinicians followed the pharmacotherapy advice recommended by DIC in their patient management. ${ }^{5}$ 


\section{Conclusion}

A smaller number of patient experiences suggests there is light at the end of the tunnel. COVID19(Coronavirus disease 2019) pandemic limited the sample size of the study. In conclusion, the maiden attempt of using pharmacology expertise in patient pharmacotherapy counseling was conducted successfully without compromising daily routine departmental work.

Acknowledgement : Authors would like to acknowledge who attended the counselling sessions at DIC.

COMPETING INTERESTS There are no competing interests to declare.

Funding : None

Data availability: Available upon request.

\section{Contributor:}

ANP and VS designed the study. CH, AK And SB collected and recoded the data. ANP and CH analyzed the data. All contributors participated in drafting the manuscript. All authors gave final approval of the version to be published. Each author participated sufficiently in the work to take public responsibility for appropriate portions of the content; and agreed to be accountable for all aspects of the work in ensuring that questions related to the accuracy or integrity of any part of the work are appropriately investigated and resolved.

\section{References:}

1. Kshirsagar N, Kumar V. Clinical pharmacology: Prospects and development in India. Indian J Pharmacol . 2011;43(5):489. doi:10.4103/0253-7613.84946

2. M PK, Patil A, Kakkar AK, Singh H. Decoding the Roadmap for Capacity Building of Pharmacology Academicians in Catering to Drug Information Center Services in a Developing Country. J Pharm Technol . 2019;35(4):146-154. doi:10.1177/8755122519841364

3. Patil AN, Padhy BM, Prasanthi SK, Rohilla R. Drug Information Center in India: Overview, Challenges and Future Prospects. Int J Pharm Investig . 2018;8(1):01-06.

4. Garg G, Patil A, Kasudhan KS. Naturalistic Evaluation of Pharmacotherapy Consultations Provided to Hospital Clinicians: A Developing Country's Perspective. J Pharm Technol . Published online April 17, 2019:8755122519843013. doi:10.1177/8755122519843013

5. Harish C, Belavigi D, Patil AN, Pattanaik S, Kakkar A, Kasudhan KS. Assessment of the Impact of Clinical Pharmacology Consultations Provided to Hospital Clinicians From the Drug Information CenterAn Outcome Research in a Developing Country. J Pharm Pract . Published online November 14, 2019:0897190019885256. doi:10.1177/0897190019885256

Figure 1: Pilot run Pharmacotherapy counseling service from Clinical Pharmacology discipline of developing world 


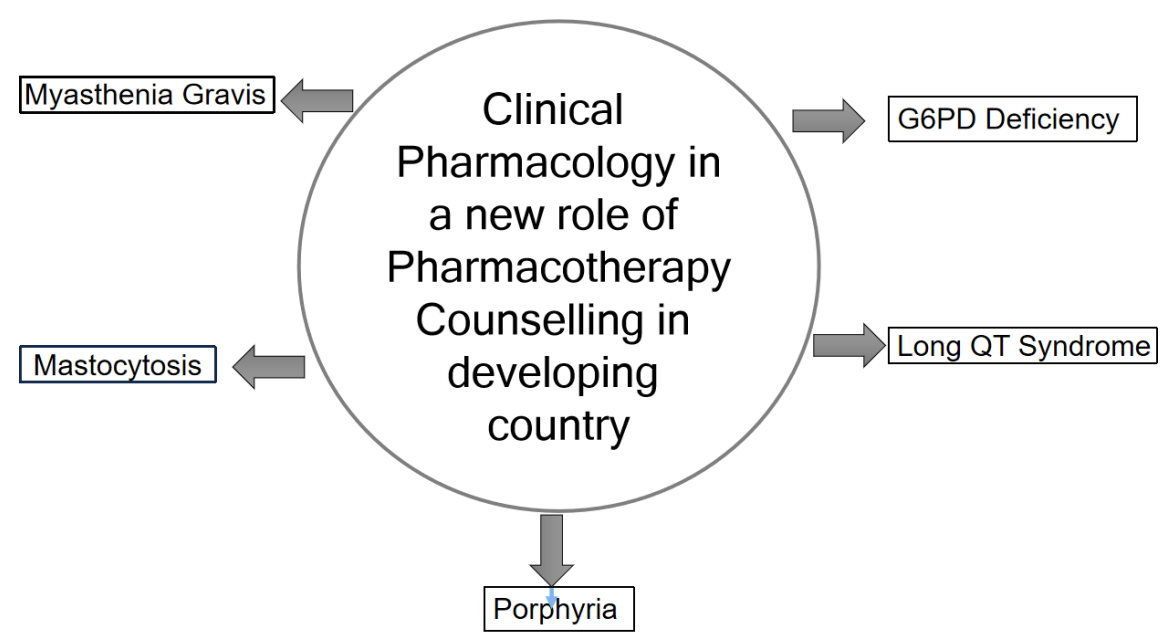

Figure 1: Pilot run Pharmacotherapy counselling service from Clinical Pharmacology discipline of developing world 